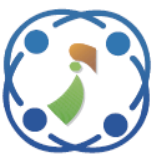

\title{
Design and FPGA Implementation of Immune-PID Controller Based on BBO Algorithm for Heart Rate Regulation
}

\author{
Eman F. Mohsin ${ }^{1} \quad$ Tariq Tashan ${ }^{2 *}$ \\ Ekhlas H. Karam ${ }^{1}$ \\ ${ }^{1}$ Computer Engineering Department, College of Engineering, Mustansiriyah University, Iraq \\ ${ }^{2}$ Electrical Engineering Department, College of Engineering, Mustansiriyah University, Iraq \\ * Corresponding author’s Email: tariq.tashan@ uomustansiriyah.edu.iq
}

\begin{abstract}
In this paper an optimal Immune-Proportional-Integral-Derivative (Immune-PID) scheme is suggested to control to the heart rate of humans and regulate it, based on Yanagihara, Noma, and Irisawa (YNI) as mathematical model of the heart rate. The parameters of the suggested immune PID controller are optimized by using BiogeographyBased-Optimization (BBO) algorithm. In addition, the proposed controller scheme is implemented using Field Programmable Gate Array (FPGA) due to its great data storage capacity, low consumption of energy, and high speed of operation. The Xilinx system generator blocks and Spartan-6 (XC6SLX45T-3FGG484) board are used due to availability with $1 \%$ utilization of hardware platform slices. The performance of the proposed controller is compared to traditional PID and immune controllers. That comparative analysis results show best improvement when using the proposed controller with $0 \%$ maximum overshoot, a reduction of steady state error and rising time reaches to 0.1 and 0.0023 second respectively and faster response reaches to 0.01 second.
\end{abstract}

Keywords: Use PID controller, Immune system, Biogeography-based optimization, YNI model, FPGA.

\section{Introduction}

Disease of the heart and blood vessels make up the majority of death causes around the world, mostly in advanced countries. There are two famous heartrelated conditions, slow heartbeat (bradycardia) and too-fast heartbeat (tachycardia), leading to fainting or shortness of breath and sometimes causing death [1]. When the heartbeat drops less than $60 \mathrm{bpm}$ it classified as Bradycardia, its signs of sickness become clear when the HR drops below than $50 \mathrm{bpm}$, and when the HR exceeds $120 \mathrm{bpm}$ is classified as tachycardia [2]. In order to treat these two cases, there are several solutions, including simple therapeutic drugs, or implant a pacemaker which is an electronic system that monitors cardiac functions, and compensate for any disorder.

Researchers have developed pacemaker control systems, especially in recent years, Aabid et al. use a traditional PID controller based on Ant-Colony Optimization algorithm to verify PID coefficients to regulate the HR [3]. Arunachalam et al. suggest a robust fractional-order PID controller for the cardiac pacemaker, which is tuned by the Ziegler-Nichols method [4]. Govind and Sekhar present a PID control system for HR regulation as pacemaker in an efficient way. It is executed via a loop consisting of an adaptive correction factor, the MIT rule, and Delta rule. The Simulation results claim that the suggested PID controller provides better performance than traditional PID controller [5]. Castroa and Malathi suggest a classical PID controller with backpropagation neural network, for heart rate regulation [6]. the paper uses Zeigler-Nichols tuning to determine the PID parameters, where it represents the output layer weights in the neural network. M. E. Karar suggests a new back-stepping adaptive controller for heart rate regulation, in dual sensor pacemaker based on radial basis function [7]. The developed controller is tested using pre-determined data of four patients heart rate during different activities. A Mamdani fuzzy proportional derivative controller is proposed in [8], to improve the cardiac pacemaker and regulate the heart rate. The controller 
represents an integration of the traditional PID. To obtain the optimal gains of the controller an optimization technique is used. The authors previously presented three structures for immune PID controller [9], to regulate the HR. The differential evolution algorithm is adopted to optimize the controller parameters, the best performance is obtained from the third structure. A modified modelfree sliding mode controller is presented in [10] for $\mathrm{HR}$ regulation in a pacemaker, the $\mathrm{BBO}$ algorithm is used to tune the parameters of the controller.

Although the mentioned controllers are claimed to have good performance, but these controllers use only PID approaches to improve the performance of the pacemaker, with few controllers used as pacemaker. There is no attempt to design or implement these controllers, FPGA to combine it as a hardware with the pacemaker. According to above reasons, the main aim of this paper is to design and implement an intelligent controller as a pacemaker for heart rate regulation, where the Immune-PID controller is used to regulate the abnormalities, which occurs in the $\mathrm{HR}$, the BBO algorithm is used to improve the controller parameters. To accomplish these objectives, YNI mathematical model is considered here, and implemented using FPGA (Spartan-605), due to its great datastorage capacity, low energy consumption, and high speed of operation. The rest of this paper is structured as follows: The YNI mathematical model of HR is presented in Section 2. The details of the Immune system are described in Section 3, while the design of the Immune-PID controller is explained in Section 4. The BBO algorithm is illustrated in Section 5. The FPGA implementation is addressed in Section 6. The proposed controller's analysis and simulation results are discussed in Section 7, while the final conclusions are listed in Section 8.

\section{YNI Heartbeat model}

The YNI mathematical model represents the action potential behavior of the Sino-Atrial cells and considered from its earliest models [1, 11]. The YNI model type is Hodgkin-Huxley, which describes standings of conductance and ionic-currents. It can simulate the action potentials of heart accurately, which based on the data of voltage-clamp, taking into consideration the relationship between current and voltage, and simulates the unprompted action potential. It contains one time-independent current called the leakage current $I_{l}$, and four time-dependent currents (potassium current $I_{k}$, hyper-polarization delayed inward current $I_{h}$, sodium current $I_{N a}$, and slow inward current $I_{s}$ ) as in Eq. (1). Fig. 1 shows an

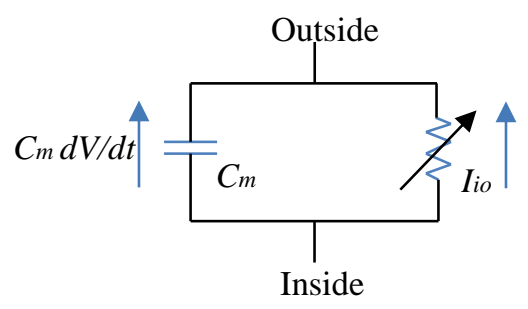

Figure. 1 The equivalent electrical circuit of cell membranes model [11]

electrical circuit equivalent to the cell membrane model. The $\mu \mathrm{A} / \mathrm{cm} 2$ is the transmembrane current unit $[11,12]$.

$$
C_{m} \frac{d V}{\mathrm{dt}}+I_{l}+I_{k}+I_{h}+I_{N a}+I_{s}=I_{a p p}
$$

where $C_{m}\left(\mu \mathrm{F} / \mathrm{cm}^{2}\right)$ refers to the cell membrane capacitance and it is a constant; $I_{a p p}$ is the externals applied current. Eq. (1) can be rewritten to be:

$$
C_{m} \frac{d V}{\mathrm{dt}}+I_{\text {ion }}=C_{m} \frac{d V}{\mathrm{dt}}+C_{m} \frac{V}{R_{m}}=I_{\text {app }}
$$

where $R_{m}$ and $C_{m}$ denote the cell membrane resistance and capacitance respectively, where:

$$
\begin{gathered}
I_{l}=0.8\left(1-\exp ^{-\frac{V+60}{20}}\right) \\
I_{k}=0.7 p \frac{\exp (0.0277(\mathrm{~V}+90))-1}{\exp (0.0277(\mathrm{~V}+40))} \\
I_{h}=0.4 q(V+45) \\
I_{N a}=0.5 m^{3} h(V-30) \\
I_{s}=12.5(0.05+0.95 d)(0.05+ \\
0.95 f)\left(\exp \frac{V-10}{15}-1\right) \\
\frac{d w}{d t}=\alpha_{w}(1-w)-\beta_{w} w
\end{gathered}
$$

and $w$ is $\{d, q, h, p, m, o r f\}$.

The gating variables $d, q, h, p, m$, and $f$ must fulfill Eq. (8) and Eq. (9) with constant values listed in Table 1, which provides the values of the constants $\alpha_{w}$ and $\beta_{w}$.

$$
\alpha_{w}, \beta_{w}=\frac{C_{1} \exp \left(\frac{\mathrm{V}-\mathrm{V} 0}{C_{2}}\right)+C_{3}(\mathrm{~V}-\mathrm{V} 0)}{1+C_{4} \exp \left(\frac{\mathrm{V}-\mathrm{V} 0}{C_{5}}\right)}
$$

The parameters $\left(\alpha_{q}, \beta_{q}, \alpha_{d}, \alpha_{p}, \beta_{f}\right)$ which do not fit Eq. (9) can be calculated by: 
Table 1. YNI model's $\alpha$ and $\beta$ values [11]

\begin{tabular}{|c|c|c|c|c|c|c|c|}
\hline & $\boldsymbol{\alpha}_{\boldsymbol{m}}$ & $\boldsymbol{\alpha}_{\boldsymbol{f}}$ & $\boldsymbol{\alpha}_{\boldsymbol{h}}$ & $\boldsymbol{\beta}_{\boldsymbol{d}}$ & $\boldsymbol{\beta}_{\boldsymbol{m}}$ & $\boldsymbol{\beta}_{\boldsymbol{p}}$ & $\boldsymbol{\beta}_{\boldsymbol{h}}$ \\
\hline$C_{1}$ & 0 & 0 & $\begin{array}{c}1.209 \times \\
10^{-3}\end{array}$ & 0 & 40 & 0 & 1 \\
\hline$C_{2}$ & - & - & -6.534 & - & -17.8 & - & $\infty$ \\
\hline$C_{3}$ & 1 & $\begin{array}{c}-3.55 \times \\
10^{-4}\end{array}$ & 0 & $\begin{array}{c}-4.21 \times \\
10^{-3}\end{array}$ & 0 & $\begin{array}{c}-2.25 \times \\
10^{-4}\end{array}$ & 0 \\
\hline$C_{4}$ & -1 & -1 & 0 & -1 & 0 & -1 & 1 \\
\hline$C_{5}$ & -10 & 5.633 & - & 2.5 & - & 13.3 & -10 \\
\hline$V_{0}$ & -37 & -20 & -20 & 5 & -62 & -40 & -30 \\
\hline
\end{tabular}

$$
\begin{gathered}
\alpha_{q}=3.4 \times 10^{-4} \frac{(\mathrm{V}+100)}{\exp \left(\frac{\mathrm{V}+100}{4.4}\right)-1}+4.95 \times 10^{-5}(10) \\
\beta_{q}=5 \times 10^{-4} \frac{(\mathrm{V}+40)}{1-\exp \left(-\frac{\mathrm{V}+40}{6}\right)}+8.45 \times 10^{-5}(11) \\
\alpha_{d}=1.045 \times 10^{-2} \frac{(\mathrm{V}+35)}{1-\exp \left(-\frac{\mathrm{V}+35}{2.5}\right)}+3.125 \times \\
\alpha_{p}=9 \times 10^{-2} \frac{V}{1-\exp \left(-\frac{\mathrm{V}}{4.8}\right)} \frac{1}{1+\exp \left(-\frac{\mathrm{V}+3.8}{9.71}\right)}+6 \times 10^{-4} \\
\beta_{f}=9.44 \times 10^{-4} \frac{(\mathrm{V}+60)}{1-\exp \left(-\frac{\mathrm{V}+29.5}{4.16}\right)}
\end{gathered}
$$

Further detailes regarding the derivation of the equations in this section, and the values of constants mentioned in Table 1, are clearly derived and addressed in [11].

\section{The immunity system}

The system of immunity is a combination of vital operations, which carried out by particles, cells, and organs distributed throughout the body, to save it from poisons, diseases, alien particles, and harmful cells. This biological system identifies and break down pathogens, like viruses or microbes. The system of immunity can recognize between the alien creatures that cause damage and the healthy body cells, which is considered the most essential function. To stimulate proper mechanisms of protections, alien cells are also categorized. The primary kind of immunity cells is the lymphocytes, which includes two main types of B lymphocytes cells that are produced in the marrow of the bone. The human body contains several different kinds, each one produces ' $\mathrm{Y}$ ' shape antibodies from their surfaces with a different chemical structure. The second type is T lymphocytes cells, which are created from the thymus, which in turn categorized into T-suppressor cells (TS) and T-helper cells (TH) [13].

Once the antigens invade the organism's body, they are identified by the Antigen Presenting Cell which sends a warning signal to the $\mathrm{T}$ cells. Upon receiving the warning, it will stimulate $B$ cells to immediately create antibodies to eliminate those

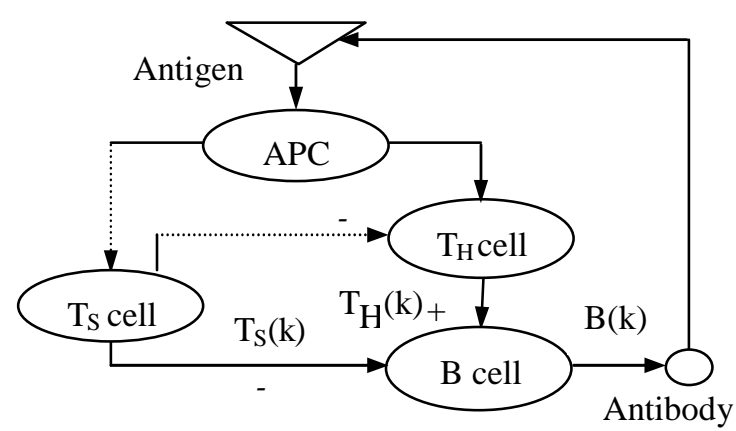

Figure. 2 Mechanism of the immunity feedback control [13]

antigens [14].

Based on the immunity feedback control mechanism shown in Fig. 2, the consistency of the Bcells can be obtained at the kth iteration as follow [13]:

$$
\begin{gathered}
B(k)=T_{H}(k)-T_{S}(k) \\
T_{H}(k)=K_{1} \delta(k) \\
T_{S}(k)=K_{2}\{f[\Delta B(k-d)]\} \delta(k)
\end{gathered}
$$

Where the interaction between the antibody that impacts the B-cells and antigen is represented by the nonlinear function $\mathrm{f}($.$) , while the consistency of the$ antigen at the kth generation is represented by $\delta(\mathrm{k})$, $\mathrm{B}(\mathrm{k})$ is the B-cell's consistency, the helper gene and the suppressor gene are represented by $\mathrm{K} 1$ and $\mathrm{K} 2$, and the consistency change of the $\mathrm{B}$-cell is represented by $\Delta \mathrm{B}(\mathrm{k}-\mathrm{td})=\mathrm{B}(\mathrm{k}-\mathrm{td})-\mathrm{B}(\mathrm{k}-\mathrm{td}-1)$, where the immune response time delay is signified by td. The relationship between the antigen and the consistency of B-cells, according to Eq. (15) - (17) can be written as:

$$
\mathrm{B}(\mathrm{k})=\mathrm{K} 1\{1-\eta \mathrm{f}[\Delta \mathrm{B}(\mathrm{k}-\mathrm{td})]\} \delta(\mathrm{k})
$$

where the proportional coefficient between TS and $\mathrm{TH}$ is represented by $\eta=\mathrm{K} 2 / \mathrm{K} 1$.

\section{The immune PID controller}

The Immune-PID is suggested here as an arrhythmia regulator. The BBO algorithm is used to compute the Immune and PID blocks parameters. The amount of antigen $\delta(k)$ is assumed as the error $e(k)$, and the output of the controller $u(k)$ is the total motivation that is accepted by the B-cells. The feedback control system is represented as:

$$
u(k)=K\left\{1-\eta f\left[\Delta u\left(k-t_{d}\right)\right]\right\} e(k)=K_{I} e(k)
$$




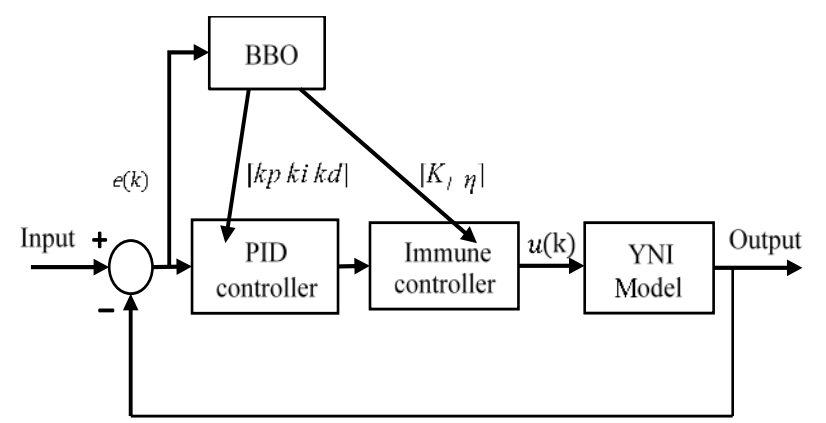

Figure. 3 The block diagram of the Immune-PID controller

Where the proportional gain of immune controller is represented by $K(1-\eta f[\Delta u(k-$ $t d)])=K_{I}$, the gene that controls the $K_{I}$ is $\eta=K_{2} / K_{1}$, and $f($.) refers to a non-linear function. $f($.$) is chosen$ here as the action function of the T-cell's regulator, based on the consistency of the antigen influence on the antibody in the response of immune.

$$
f(x)=\frac{1}{1+\exp (-c x)} \quad, \quad c>0
$$

Where $c$ has a positive value that determines the active region of $x$, the range of $f(x)$ is [0-1]. The output of the immune controller can be written as:

$u(k)=K\left\{1-\eta\left(\frac{1}{1+\exp (-c x)}\right)\right\} e(k)=K_{I} e(k)(21)$

The traditional PID must be combined with the immune controller, because the immune controller is a nonlinear proportional controller. Therefore, it is not robust against the error caused by non-linear disturbance or against noise. The result of this combination can improve the system's performance and overcome this problem as shown in Fig. 3 [13$15]$.

$$
\begin{gathered}
u(k)=k_{I} \times k_{p}\left[1+\frac{k_{i}}{1-z^{-1}}+k_{d}\left(1-z^{-1}\right)\right] e(k)= \\
k_{I P}\left[1+\frac{k_{i}}{1-z^{-1}}+k_{d}\left(1-z^{-1}\right)\right] e(k)
\end{gathered}
$$

where $k_{I P}$ represents the proportional gain.

\section{BBO Algorithm}

The BBO algorithm is based on the improvement of biogeography, it describes in a mathematical way, the migration of species from one island to another, this migration targets better places to live, the algorithm is proposed in 2008 by D. Simon [16]. The transitions of species from one island to another in biogeography depend on Habitat Suitable Index
(HSI), which in turn depends on several characteristics such as the diversity of food cover, temperature, land area, and water resources. These characteristics are called Suitability-Index-Variables (SIV). HSI is similar to the cost or fitness function in other algorithms of population-based optimization and SIV point out the tuned parameter's number [17]. Generally, two steps are included in the BBO algorithm: information sharing (migrations) and mutation.

\subsection{Migrations}

The habitat with the highest HSI is the best for living species, therefore, the migration of species between habitats occurs when the HSI of habitats is low, or if more species congregate in the same habitat. The previous process is called Emigration. When the species migrate to a habitat with high HSI and few species, the process is called Immigration. Both Emigration and Immigration are also called migration $[18,19]$. The process of migration used to change the solution $H_{i}$ by involving the properties from another solution $H_{j}$, which can be determined via:

$$
H_{i}\left(S I V_{k}\right) \leftarrow H_{j}\left(S I V_{k}\right)
$$

An $H_{i}$ solution emigration or immigration can be determined according to the emigration rate $\mu_{i}$ and immigration rate $\lambda_{i}[17] . \mu_{i}$ and $\lambda_{i}$ are calculated using Eq. (24) and Eq. (25), in each habitat which have a different value.

$$
\begin{gathered}
\mu_{i}=\frac{k_{i}}{n} E \\
\lambda_{i}=\left(1-\frac{k_{i}}{n}\right) I
\end{gathered}
$$

Where $I$ and $E$ represent the maximum immigration rate $\lambda$ and the maximum emigration rate $\mu$ respectively, $n$ denotes the population size, $k_{i}$ is the rank $H_{i}$ after evaluation.

\subsection{Mutations}

The probabilistic factor (mutation) is used to add new features to a randomly selected solution via modifying one or more of its SIVs, based on $P_{i}$ which increases the diversity of population [16], Eq. (26) illustrates the mutation of the $i_{t h}$ habitat.

$$
M_{i}=m_{\max }\left(1-\frac{P_{i}}{P_{\max }}\right)
$$




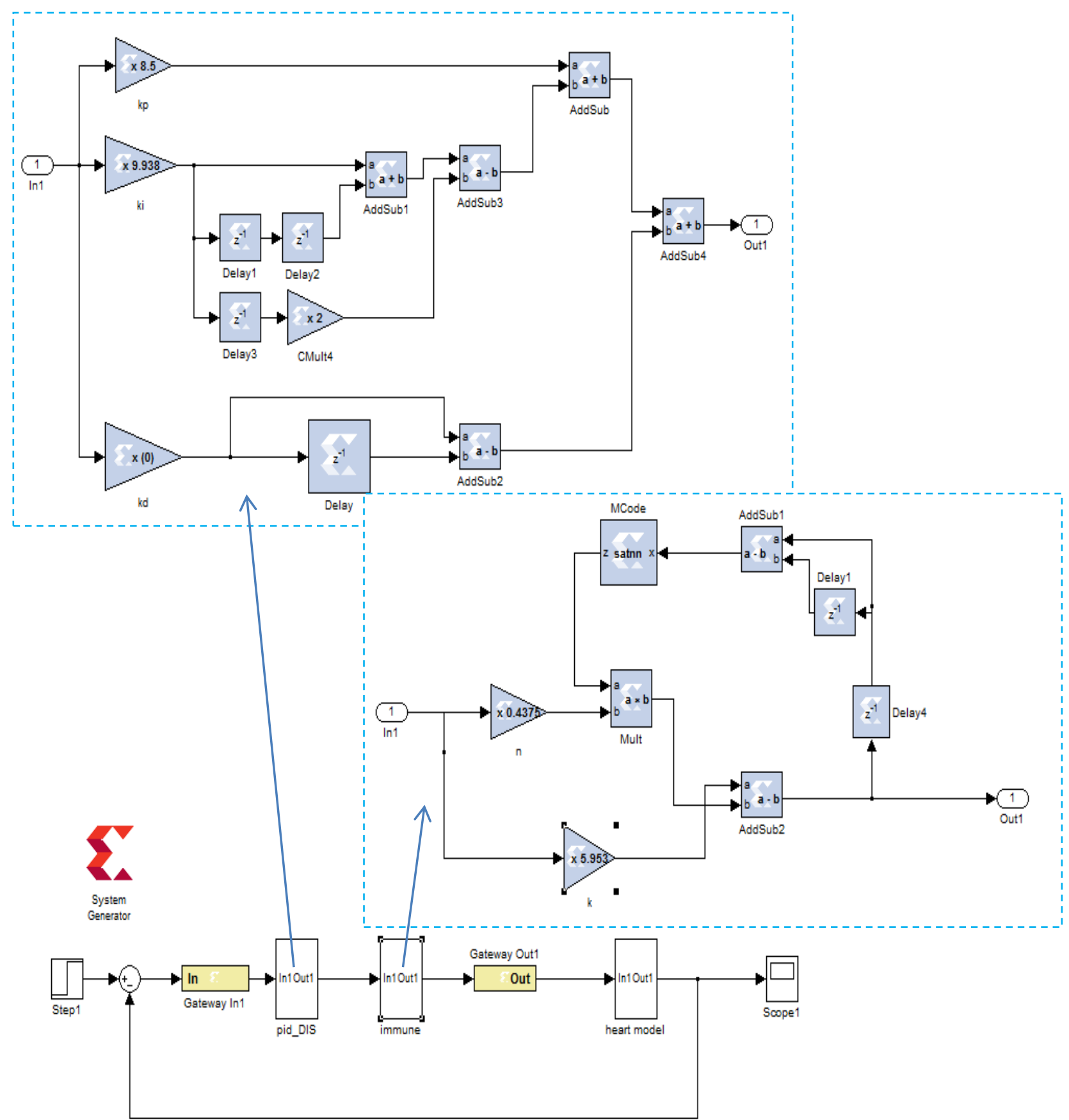

Figure. 4 FPGA design of immune-PID controller

Where $P_{i}$ is the existence probability of the habitat $H_{i}$ and $m_{\max }$ is defined by the user and represents the mutation coefficient.

\section{FPGA implementation}

The libraries of the Xilinx System Generator block set [20], are used here to execute the immunePID controller. In order to implement this controller using FPGA, a Spartan-6 (XC6SLX45T-3FGG484) FPGA Kit is suggested. In this paper the" saturation function" is used instead of the non-linear function $f(x)$ in the immune part of the proposed controller, due to the difficulty of implementation in FPGA [21]. In order to produce the best resolution, the input signal is represented using fixed-point sign number of 10 bits, with 3 bits binary points (Fix 103 ), while the output signal is represented using (Fix 48 26). Fig. 4 and Fig. 5 show the FPGA design and the device utilization summary of the immune-PID controller with $1 \%$ utilization of hardware platform slices respectively. To interface the hardware platform with MATLAB Simulink, JTAG cable is used to configure the suggested controller into the FPGA kit Fig. 6 illustrates this process. 


\begin{tabular}{|c|c|c|c|c|c|}
\hline & evice Utilization Summ & & & & {$[-]$} \\
\hline Slice Logic Utilization & Used & Available & Utilization & Note(s) & \\
\hline Number of Slice Registers & 138 & 54,576 & $1 \%$ & & \\
\hline Number used as Flip Flops & 131 & & & & \\
\hline Number used as Latches & 0 & & & & \\
\hline Number used as Latch-thrus & 0 & & & & \\
\hline Number used as AND/OR logics & 7 & & & & \\
\hline Number of Slice LUTs & 351 & 27,288 & $1 \%$ & & \\
\hline Number used as logic & 332 & 27,288 & $1 \%$ & & \\
\hline Number using 06 output only & 269 & & & & \\
\hline Number using 05 output only & 32 & & & & \\
\hline Number using 05 and 06 & 31 & & & & \\
\hline Number used as ROM & 0 & & & & \\
\hline Number used as Memory & 0 & 6,408 & $0 \%$ & & \\
\hline Number used exclusively as route-thrus & 19 & & & & \\
\hline Number with same-slice register load & 8 & & & & \\
\hline Number with same-slice carry load & 11 & & & & \\
\hline Number with other load & 0 & & & & \\
\hline Number of occupied Slices & 127 & 6,822 & $1 \%$ & & \\
\hline Number of MUXCYs used & 304 & 13,644 & $2 \%$ & & \\
\hline Number of LUT Flip Flop pairs used & 393 & & & & \\
\hline Number with an unused Flip Flop & 263 & 393 & $66 \%$ & & \\
\hline Number with an unused LUT & 42 & 393 & $10 \%$ & & \\
\hline Number of fully used LUTFF pairs & 88 & 393 & $22 \%$ & & \\
\hline Number of unique control sets & 1 & & & & \\
\hline $\begin{array}{l}\text { Number of slice register sites lost } \\
\text { to control set restrictions }\end{array}$ & 5 & 54,576 & $1 \%$ & & \\
\hline
\end{tabular}

Figure. 5 The device utilization summary of the immune-PID controller

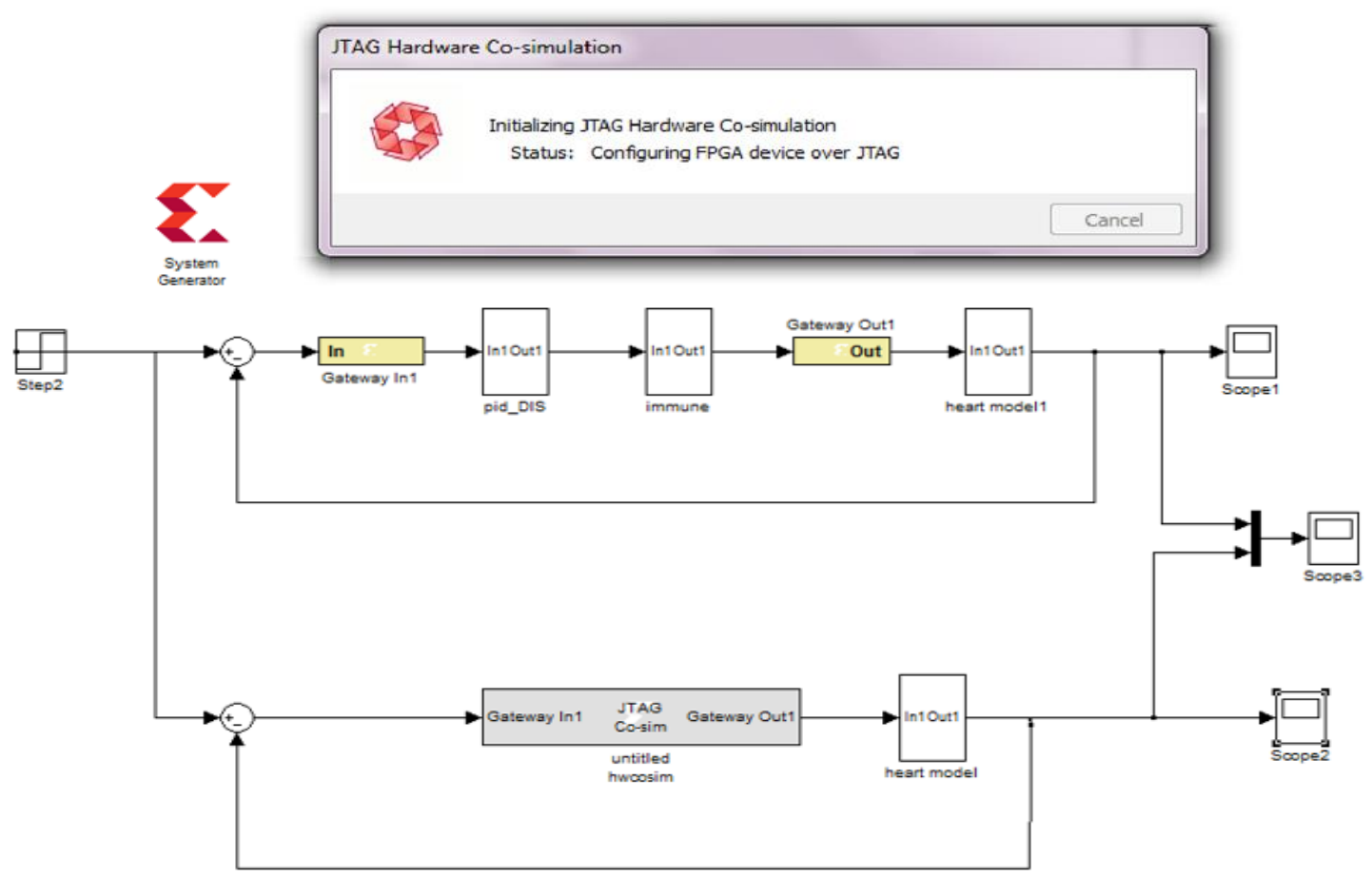

Figure. 6 Downloading the bit stream to the hardware device through JTAG cable

\section{Simulation results}

The results of simulation for the YNI heart model which addressed in Eq. (1), and the suggested controller based on the BBO algorithm are offered in this section for an HR of $70 \mathrm{bpm}$. The YNI model response without controller is shown in Fig. 7.

The parameters of the $\mathrm{BBO}$ algorithm are considered here as in Table 2.

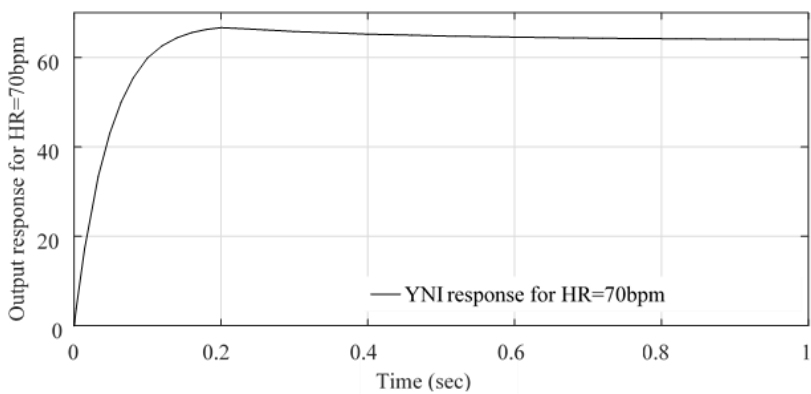

Figure. 7 The response of the YNI heart model for HR= $70 \mathrm{bpm}$ 
Table 2. The parameters of the BBO algorithm.

\begin{tabular}{|c|c|}
\hline BBO parameter & Value \\
\hline Generation number & 10 \\
\hline Mutation Probability & 0.06 \\
\hline n (Habitats) & 20 \\
\hline SIV & 5 \\
\hline E & 1 \\
\hline I & 1 \\
\hline Min Domain & 0 \\
\hline Max Domain & 10 \\
\hline
\end{tabular}

Table 3. Optimal controller parameters.

\begin{tabular}{|l|l|l|l|}
\hline Parameter & Immune & PID & Immune-PID \\
\hline$k_{p}$ & & 3.6085 & 8.531 \\
\hline$k_{i}$ & & 6.6866 & 9.937 \\
\hline$k_{d}$ & & 0.0027 & 0.0003 \\
\hline$K_{1}$ & 7.9005 & - & 5.9601 \\
\hline$c$ & 0.5 & - & 0.5 \\
\hline$\eta$ & 0.1567 & & 0.4424 \\
\hline
\end{tabular}

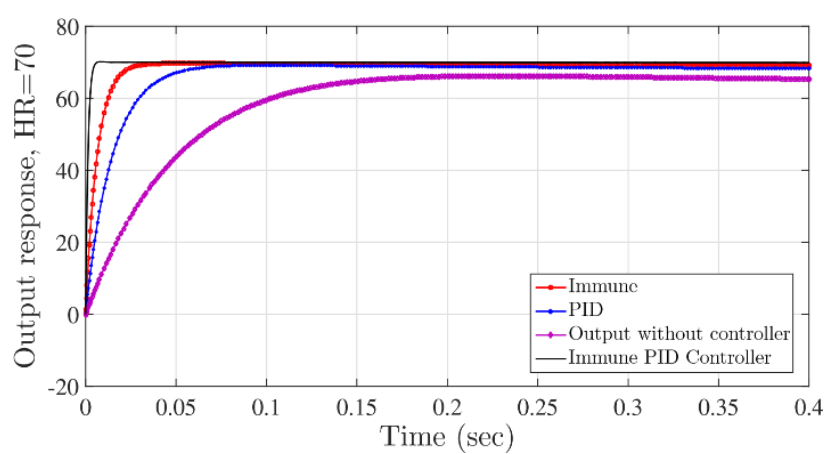

Figure. 8 The YNI model response regulated by immune, PID, and immune-PID controllers for $\mathrm{HR}=70$ bpm

Table 3 illustrates the optimal obtained parameters for the Immune, classical PID, and Immune-PID controllers from the BBO algorithm.

Fig. 8 shows the response of the YNI model after applying the suggested controllers to regulate the HR, using the parameters in Table 3.

It can be noticed from Fig. 8 that the cardiac system with these controllers tracks the desired HR with small rise time $\left(t_{r}\right)$, settling time $\left(t_{s}\right)$, and study state error (e.s.s.).

The comparison between all controllers is shown in Table 4. This table illustrates the performance of the three controllers. The immune-PID has the best average performance with $\left(M_{p}=0 \%, t_{s}=0.01 \mathrm{sec}, t_{r}\right.$ $=0.0023 \mathrm{sec}$ and $e_{s s}=0.1$ ) which satisfies the design requirement. While the other methods of the PID tuned by BBO, the PID and the immune controllers provide a plausible response. Table 4 addresses a direct comparison between the three controllers, and Robust RBF-neural-network based backstepping
Table 4. Optimal controller parameters

\begin{tabular}{|l|l|l|l|l|}
\hline Parameter & PID & Immune & $\begin{array}{l}\text { Immune- } \\
\text { PID }\end{array}$ & $\begin{array}{l}\text { RBF- } \\
\text { neural- } \\
\text { network[7] }\end{array}$ \\
\hline$M_{p}(\%)$ & 0 & 0 & 0 & 1.6 \\
\hline$t_{r}(\mathrm{sec})$ & 0.0169 & 0.0135 & 0.0023 & 0.155 \\
\hline$t_{s}(\mathrm{sec})$ & 0.05 & 0.04 & 0.01 & 0.244 \\
\hline ess & 1.03 & 0.8 & 0.1 & 1.60 \\
\hline
\end{tabular}

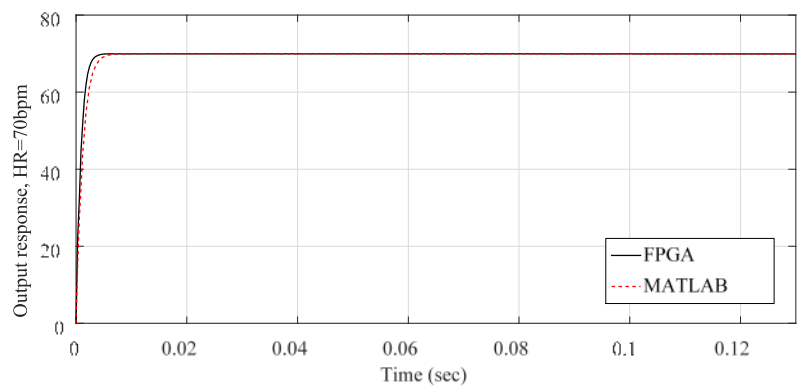

Figure. 9 The YNI model response with $\mathrm{HR}=70 \mathrm{bpm}$ regulated by immune-PID controller in FPGA

control in [7] with respect to the maximum overshoot $(\%)$, rise time, settling time and ess respectively.

Fig. 9 illustrates the simulation results of the controlled cardiac system in MATLAB and FPGA. To evaluate the robustness of the proposed controller against random variable input, an HR in the range of $(70 \pm 10) \mathrm{pbm}$ is considered, then a sudden disturbance is applied to mimic the sudden Tachycardia, which represented by adding step function with amplitude of 40 at the $5^{\text {th }}$ second without removing it. A sudden bradycardia scenario is also evaluated by adding a step function with amplitude of -30 at the $5^{\text {th }}$ second without removing it. The results are similar in both Matlab and FPGA, but little chatter is found in the outperformance of the FPGA caused by the use of a fixed point.

Fig. 10 shows a comparison between the simulated performance of FPGA and Matlab. Where Fig. 10 (a) shows the response of the heart of tracking a random input without external influence on it. While Fig. 10 (b) illustrates its rapid response against Tachycardia, where the proposed controller manages to return to the natural range of HR and overcome this rise within only $(0.0185 \mathrm{sec}$.). The proposed controller is also robust against the sudden Bradycardia and can overcome it and regulate the HR within only (0.0106 sec.) as shown in Fig. 10 (c).

\section{Conclusions}

In this paper instead of using conventional discrete PID, the Immune-PID controller is suggested for human heart rate regulation. The YNI heart mathematical model is considered here. The 


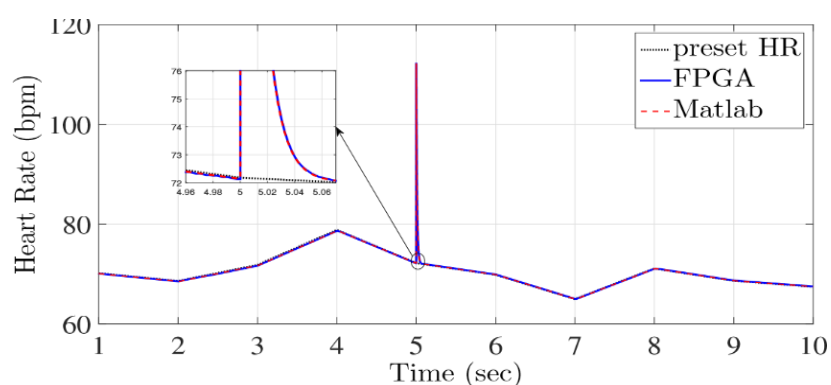

(a)

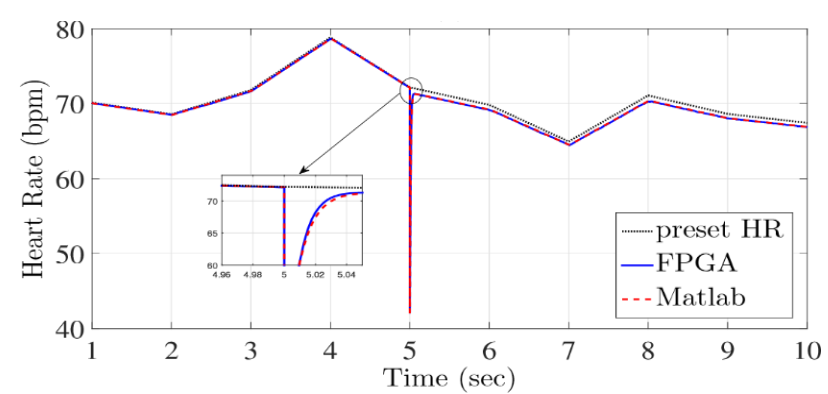

(b)

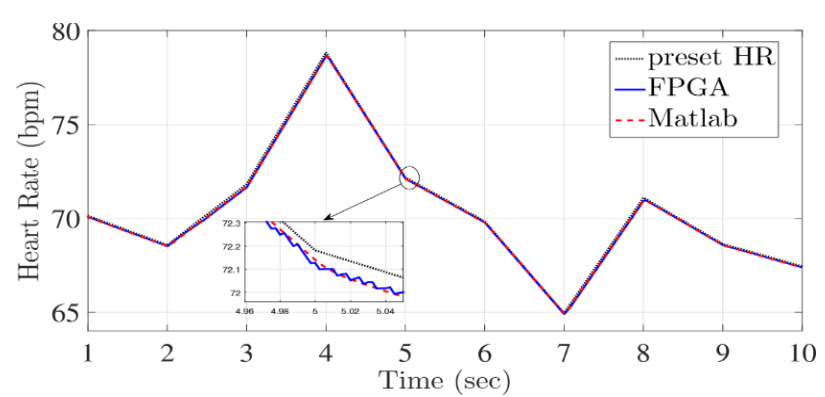

(c)

Figure. 10 The HR at rest where: (a) the normal case, (b) with tachycardia, and (c) with bradycardia.

suggested controller optimal parameters are determined using the BBO algorithm. In addition, the optimal Immune-PID controller is implemented using FPGA due to its great data storage capacity, low consumption of energy, and high speed of operation in order to design this controller as a simple Pacemaker circuit. According to the simulation results, the immune-PID controller outperforms the PID, Immune controllers and backstepping controller [7] in terms of ts, tr, and e.s.s as shown in Table 4. The proposed controller shows significant robustness in overcoming sudden changes in the HR, for the two scenarios (Tachycardia and Bradycardia), as shown in Fig. 10, the controller successfully returns the HR to its normal range within less than $20 \mathrm{msec}$. in each case.

\section{Conflicts of Interest}

The authors declare no conflict of interest.

\section{Author Contributions}

The Algorithm development, methodology, Matlab simulation, FPGA implementation, early draft writing, and results were provided by Eman F. Mohsin. Tariq Tashan and Ekhlas H. Karam jointly directed and supervised the research, reviewed, revised the paper.

\section{Acknowledgments}

The Authors would like to thank all colleagues at Computer Engineering Department, College of Engineering, Mustansiriyah University for their support.

\section{References}

[1] W. V. Shi and M. Zhou, "Optimal single-pulse for pacemakers based on a sinoatrial model", IEEE/ASME Transactions on Mechatronics, Vol. 18, No. 1, pp. 348-354, 2013.

[2] K. P. Anderson, "Sudden cardiac death unresponsive to implantable defibrillator therapy: an urgent target for clinicians, industry and government", 2005.

[3] M. Aabid, A. Elakkary, and N. Sefiani, "Pid parameters optimization using ant-colony algorithm for human heart control", In: Proc. of 2017 23rd International Conf. on Automation and Computing (ICAC). IEEE, pp. 1-6, 2017.

[4] S. P. Arunachalam, S. Kapa, S. K. Mulpuru, P. A. Friedman, and E. G. Tolkacheva, "Intelligent fractional-order pid (fopid) heart rate controller for cardiac pacemaker", In: Proc. of 2016 IEEE Healthcare Innovation Point-Of-Care Technologies Conf. (HI-POCT), pp. 105-108, 2016.

[5] K. A. Govind and R. A. Sekhar, "Design of a novel pid controller for cardiac pacemaker", In: Proc. of 2014 International Conf. on Advances in Green Energy (ICAGE), pp. 82-87, 2014.

[6] V. J. F. Castro and R. Malathi, "Design of advanced control strategies for cardiovascular system", Materials Today: Proc., Vol. 5, No. 1, pp. 1960-1966, 2018.

[7] M. E. Karar, "Robust rbf neural network-based backstepping controller for implantable cardiac pacemakers", International Journal of Adaptive Control and Signal Processing, Vol. 32, No. 7, pp. 1040-1051, 2018.

[8] M. I. Elnaggar, A. S. Ashour, Y. Guo, H. A. ElKhobby, and M. M. A. Elnaby, "An optimized mamdani fpd controller design of cardiac pacemaker", Health Information Science and Systems, Vol. 7, No. 1, p. 2, 2019. 
[9] T. Tashan, E. H. Karam, and E. F. Mohsin, "Immune pid controller based on differential evolution algorithm for heart rate regulation", International Journal of Advanced Computer Research, Vol. 9, No. 42, pp. 177-185, 2019.

[10] E. H. Karam, T. Tashan, and E. F. Mohsin, "Design of model free sliding mode controller based on bbo algorithm for heart rate pacemaker", International Journal of Modern Education and Computer Science, Vol. 11, No. 3, p. 31, 2019.

[11] J. P. Keener and J. Sneyd, Mathematical physiology.Springer, Vol. 1, 1998.

[12] K. Yanagihara, A. Noma, and H. IRISAWA, "Reconstruction of sino-atrial node pacemaker potential based on the voltage clamp experiments", The Japanese Journal of Physiology, Vol. 30, No. 6, pp. 841-857, 1980.

[13] W. Wang, X. Z. Gao, and C. Wang, "A new immune pid controller in material-level control", In: Proc. of Third International Conf. on Natural Computation (ICNC 2007), Vol. 3, pp. 614-618, 2007.

[14] X.-h. Liu, X.-h. Chen, X.-h. Zheng, S.-p. Li, and Z.-b. Wang, "Development of a ga-fuzzyimmune pid controller with incomplete derivation for robot dexterous hand", The Scientific World Journal, Vol. 2014, 2014.

[15] W. Wang, X. Gao, and C. Wang, "Fuzzy immune pid controller in material-level control of preheating cylinder", In: Proc. of International Conf. on Informatics and Control Technologies, pp. 52-55, 2006.

[16] D. Simon, "Biogeography-based optimization", IEEE Transactions on Evolutionary Computation, Vol. 12, No. 6, pp. 702-713, 2008.

[17] M. Salem and M. F. Khelfi, "Application of biogeography based optimization in tuning a pid controller for nonlinear systems", In: Proc. of 2012 IEEE International Conf. on Complex Systems (ICCS), pp. 1-6, 2002.

[18] H. Ma, D. Simon, P. Siarry, Z. Yang, and M. Fei, "Biogeography-based optimization: a 10-year review", IEEE Transactions on Emerging Topics in Computational Intelligence, Vol. 1, No. 5, pp. 391-407, 2017.

[19] K. Rajagopal and L. Ponnusamy, "Biogeography-based optimization of pid tuning parameters for the vibration control of active suspension system", Journal of Control Engineering and Applied Informatics, Vol. 16, No. 1, pp. 31-39, 2014.

[20] System generator for dsp getting started guide, 2012.
[21] T. Saidani, D. Dia, W. Elhamzi, M. Atri, R. Tourki et al., "Hardware co-simulation for video processing using xilinx system generator", In: Proc. of the World Congress on Engineering, Vol. 1, pp. 3-7, 2009. 